\title{
Probing Noncovalent Protein-Ligand Interactions of the cGMP-Dependent Protein Kinase Using Electrospray Ionization Time of Flight Mass Spectrometry
}

\author{
Martijn W. H. Pinkse and Albert J. R. Heck \\ Department of Biomolecular Mass Spectrometry, Bijvoet Center for Biomolecular Research and Utrecht \\ Institute for Pharmaceutical Sciences, Utrecht University, Utrecht, The Netherlands
}

Klaus Rumpel and Frank Pullen

Pfizer Global Research and Development, Sandwich, United Kingdom

\begin{abstract}
Nanoflow electrospray ionization time of flight mass spectrometry (ESI-TOF-MS) was used to study activation properties of the cGMP-dependent protein kinase (PKG). Our nanoflow ESI-TOF-MS analysis confirms that PKG mainly occurs as a $153 \mathrm{kDa}$ homodimer and is able to bind four cGMP molecules, which is in agreement with the known stoichiometry. Binding order and stoichiometry of cGMP, the non-hydrolysable ATP analog $\beta, \gamma$-imidoadenosine $5^{\prime}$-triphosphate (AMPPNP) and $\mathrm{Mn}^{2+}$ for PKG were characterized as model for the active PKG-cGMP-ATP $/ \mathrm{Mg}^{2+}$ complex. Already in the absence of cGMP, a noncovalent complex between PKG and two molecules of AMPPNP could be observed by ESI-TOF-MS. Binding of AMPPNP to PKG was strongly enhanced by the addition of $\mathrm{MnCl}_{2}$ to the spray solution. This is in agreement with binding of AMPPNP $/ \mathrm{Mn}^{2+}$ in the ATP binding pocket of PKG since all protein kinases require a metal ion to accompany ATP in the ATP-binding pocket for proper positioning of the $\beta$ and $\gamma$ phosphates. Additionally, this finding could imply that within the inactive conformation of PKG, the autoinhibition-domain, when in contact with the substratedocking domain, does not block the entrance to the ATP-binding site. In the presence of cGMP, less of the fully saturated PKG-(cGMP $)_{4}\left(\mathrm{AMPPNP} / \mathrm{Mn}^{2+}\right)_{2}$ complex was observed, suggesting that the PKG-ATP interaction is weakened in the active conformation of PKG. Additionally, limited proteolysis in combination with native-ESI MS showed to be a useful tool to study the contact regions on the PKG-dimer and also allowed the rapid determination of the overall autophosphorylation status of the protein. These measurements indicated that autophosphorylation mainly occurs within the first 80 aminoterminal residues and involves in total 3-4 phosphates per subunit. (J Am Soc Mass Spectrom 2004, 15, 1392-1399) (c) 2004 American Society for Mass Spectrometry
\end{abstract}

$\mathrm{P}$ rotein kinases are members of a huge family of enzymes that share together with protein phosphatases the responsibility of regulating virtually every kind of cellular function. Activation of protein kinases is one of the major mechanisms by which cellular events are controlled. Hence, intense effort is presently directed towards understanding the molecular basis and mechanisms of protein kinases. Protein kinases serve as molecular switches and are thus by definition highly dynamic proteins that can toggle

Published online August 25, 2004

Address reprint requests to Dr. M. W. H. Pinkse, Department of Biomolecular Mass Spectrometry, Bijvoet Center for Biomolecular Research and Utrecht Institute for Pharmaceutical Sciences, Utrecht University, Sorbonnelaan 16, 3584 CA Utrecht, The Netherlands. E-mail: m.w.h.pinkse@ pharm.uu.nl between different conformational states. Most protein kinases are also phosphoproteins, and such phosphates are often an integral determinant for both structure and function. Crystal structures of several kinases revealed that the active forms of these enzymes show similar conformations in key regions, but their inactive conformations exhibit surprisingly large structural variations [1]. Within the large and diverse family of protein kinases, the cAMP dependent protein kinase (PKA) is one of the simplest and best understood members and often serves as a prototype for the entire family [2-4]. PKA is a heterotetramer composed of a regulatory dimer and two catalytic subunits. The catalytic subunits are active when cAMP binds to the regulatory dimer and induces dissociation of the tetramer. The catalytic subunit of PKA is comprised of a bilobal core that is 
shared by all members of the protein kinase family. The two lobes, which are connected via a small linker region, generate a binding pocket for ATP and a docking site for protein/peptide substrates. The ATP is nestled deep between the two domains, while substrate peptides/proteins dock onto the more exposed region of the larger C-terminal lobe. Protein kinases do not phosphorylate free amino acids efficiently and they rely partly on local residues within the substrate for high affinity. Subtle differences within the peptide-docking domain in the large lobe of the catalytic core of each kinase play an important role in defining substrate specificity. In order to understand the role of these kinases in signaling pathways it is important to gain deeper insight into how specificity and different mechanisms of control within the common protein kinase fold are regulated. In general, regulation of protein kinases is achieved through phosphorylation/dephosphorylation, binding of second messengers, interaction with regulatory domains or subunits and/or assembly with accessory proteins. In the present study we have used mass spectrometry to study structural and biochemical aspects of the cGMP-dependent protein kinase (PKG) I $\alpha$. PKG is involved in the nitric oxide/cGMPsignaling pathway, serves as a major receptor protein for intracellular cGMP and controls a variety of cellular responses, including smooth muscle relaxation $[5,6]$. PKG is the closest homolog of PKA and in particular the catalytic domains of the two enzymes show a remarkable high sequence homology [7]. No detailed structural information for PKG is yet available, but on basis of biochemical data and by comparing the functional domains of PKG (Figure 1a) with PKA a simplified structure of PKG has evolved which is schematically depicted in Figure $1 b$. PKG I $\alpha$ exists as a dimer of two identical subunits $\left(\mathrm{M}_{\mathrm{r}} \sim 76 \mathrm{kDa}\right)$. Each subunit possesses two functional moieties, a regulatory and a catalytic. The $\mathrm{N}$ terminal regulatory domain contains a leucine/isoleucine dimerization domain, an autoinhibitory domain, including autophosphorylation sites and a hinge region, which connects the aminoterminus with the two in-tandem cGMP binding pockets. The catalytic domain contains the MgATP binding pocket and the peptide/protein-substrate docking site. In the inactive state of the protein, components of the inhibitory domain in the N-terminal part of the protein interact with the substrate-docking domain. Binding of cGMP induces a conformational change in PKG that modulates this interaction and this leads to activation of the enzyme [8]. Once activated by cGMP, PKG phosphorylates target substrates and it autophosphorylates residues within the inhibitory domain $[4,9,10]$. Extensive autophosphorylation of PKG in the presence of either cGMP or cAMP increases basal kinase activity. Furthermore, it has been shown that both cGMP-binding and autophosphorylation apparently alter the protein in a similar manner [10]. In the present study we have examined cGMP and ATP binding processes of (a)

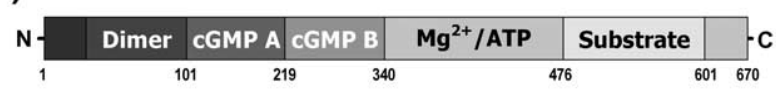

(b)

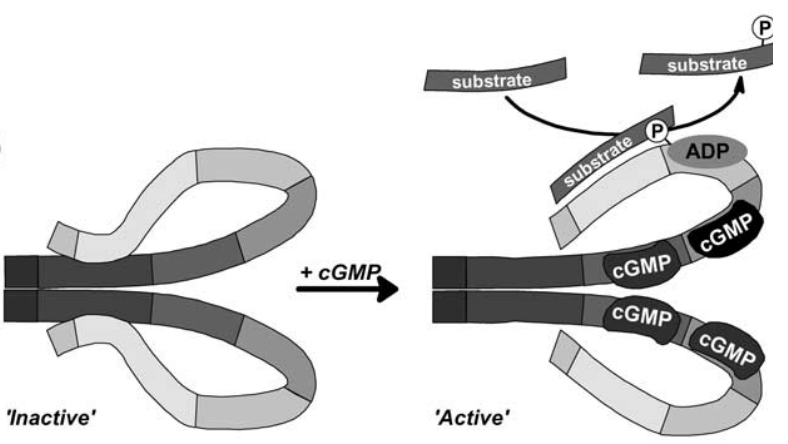

Figure 1. Schematic representation of the structure and the activation process of PKG. (a) Linear rearrangement of the functional domains of PKG. The N-terminal regulatory domain contains a leucine/isoleucine dimerization domain, an autoinhibitory domain, including autophosphorylateion sites and a hinge region, which connects the aminoterminus with the two in-tandem cGMP binding pockets (Site A, high affinity, slow dissociation; and Site $\mathrm{B}$, low affinity, rapid dissociation). The catalytic domain contains the MgATP binding pocket and the peptide/protein-substrate docking site. (b) Schematic representation of the current working model of cGMP-induced activation of PKG. Dimerization occurs via the N-terminal leucine/isoleucine zipper motif. Autoinhibition of enzymatic activity involves an interaction between the catalytic domain and the N-terminal autoinhibition domain. cGMP-binding induces a conformational change which releases PKG from its inactive conformation.

PKG I $\alpha$ by mass spectrometry based techniques. Recent examples have shown that electrospray ionization under non-denaturing conditions can provide detailed information about protein-ligand complexes, including binding order and stoichiometry of binding partners, binding affinity and cooperativity [11-14]. In this study nanoflow ESI-TOF-MS allowed the detection of noncovalent interactions between PKG, the high affinity ligand cGMP and the ATP substrate-analog AMPPNP. Our nanoflow ESI-TOF-MS analysis revealed that dimeric PKG binds four cGMP molecules, thus confirming the known PKG/cGMP stoichiometry of two cGMPs per monomer [15]. The noncovalent complex between PKG and two AMPPNP molecules could be observed already in the absence of cGMP and only in the presence of the divalent metal $\mathrm{Mn}^{2+}$. Additionally, we examined the phosphorylation state of the protein after incubation with $\mathrm{Mg}^{2+}$ and ATP at a global level. PKG I $\alpha$ is labile to specific proteolysis [16]. Limited proteolysis of the kinase with chymotrypsin yields two large fragments and an additional small peptide. Monitoring for $80 \mathrm{Da}$ mass-increments of these fragments along the autophosphorylation process by nano-ESITOF-MS allowed further insight into the overall amount of phosphate incorporation into the different parts of the kinase. 


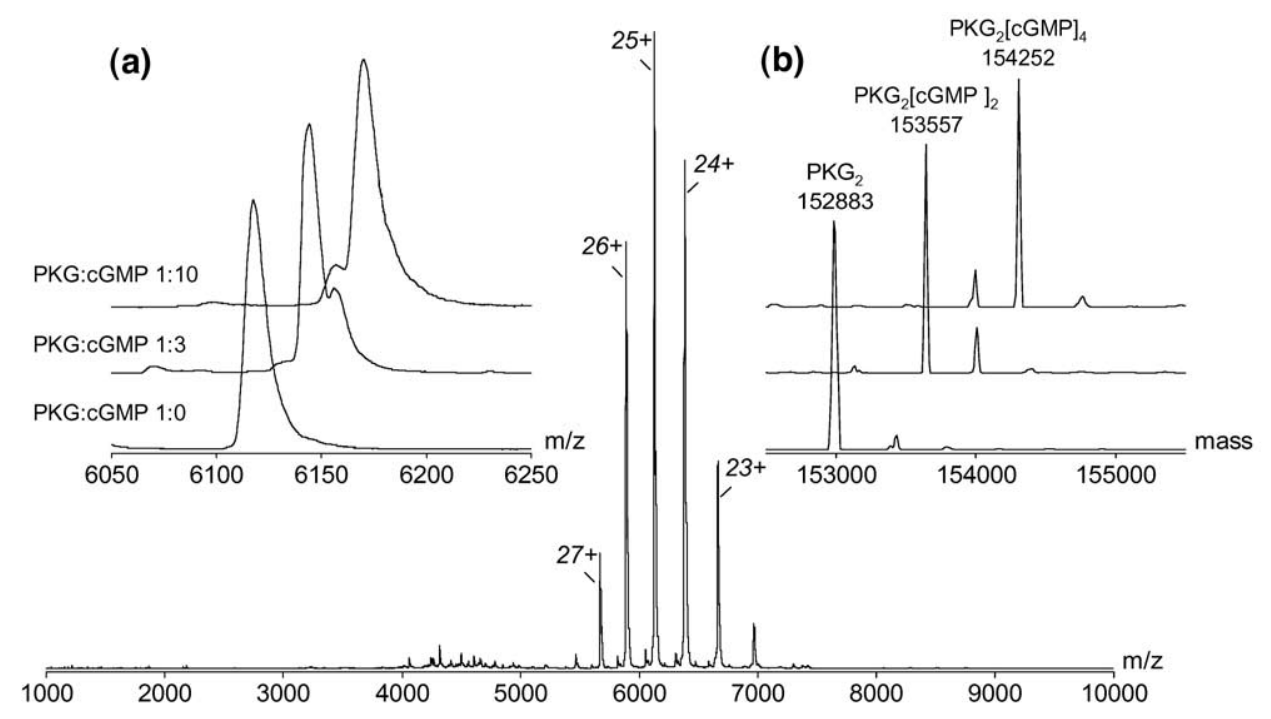

Figure 2. Electrospray ionization mass spectrum of native PKG electrosprayed from an aqueous 200 $\mathrm{mM}$ ammonium acetate solution, $\mathrm{pH} 6.7$, at an estimated dimer concentration of $\sim 1-2 \mu \mathrm{M}$. Inset (a) shows the changes of the $[\mathrm{M}+25 \mathrm{H}]^{25+}$ signal of dimeric PKG upon addition of cGMP. Inset $(\mathbf{b})$ shows he corresponding deconvoluted (MaxEnt1) mass spectra of PKG acquired in absence or presence of cGMP.

\section{Experimental}

\section{Material and Reagents}

Bovine lung PKG type I $\alpha$ was purified to homogeneity essentially as described by Francis et al. [17]. Recombinant PKG I $\alpha$ was expressed and purified from SF9insect cells according to Dostmann et al. [18]. Guanosine $3^{\prime}, 5^{\prime}$-cyclic monophosphate, Adenosine 5'-triphosphate sodium salt, $\beta, \gamma$-imidoadenosine $5^{\prime}$-triphosphate tetralithium salt, magnesium acetate tetrahydrate and manganese(II) chloride were purchased from Sigma (St. Louis, $\mathrm{MO}$ ). Sequencing grade chymotrypsin was purchased from Roche Diagnostics GmbH (Mannheim, Germany).

\section{Sample Preparation}

Prior to mass spectrometric measurements PKG was buffer exchanged to $200 \mathrm{mM}$ ammonium acetate, $\mathrm{pH} 6.7$ using Ultrafree- 0.5 centrifugal filter units (5000 NMWL) (Millipore, Bedford MA). cGMP, AMPPNP, and magnesium acetate or manganese chloride were dissolved in $200 \mathrm{mM}$ ammonium acetate, $\mathrm{pH}$ 6.7, and added to a recombinant PKG solution $(\sim 5 \mu \mathrm{M})$. For the preparation of differentially phosphorylated protein, PKG isolated from bovine lung was incubated with ATP and $\mathrm{MgCl}_{2}$. Non-phosphorylated protein was not incubated with $\mathrm{MgCl}_{2}$ and ATP. In order to obtain partially- and highly-autophosphorylated protein, PKG was incubated with $5 \mathrm{mM} \mathrm{MgCl}_{2}$ and $100 \mu \mathrm{M}$ ATP at $30{ }^{\circ} \mathrm{C}$ for 10 min and $3 \mathrm{~h}$, respectively. Limited proteolysis of autophosphorylated PKG with chymotrypsin was performed as essentially described by Monken et al. [16]. The protein/chymotrypsin ratio used was 1:200 by weight.

\section{Electrospray Ionization Mass Spectrometry}

Electrospray ionization mass spectrometry analyses of the intact protein were carried out on a Micromass LC-T TOF instrument (Micromass, Manchester, UK) equipped with a "Z-Spray" nanoflow electrospray source using in-house pulled and gold coated borosilicate glass needles. Typical ESI-TOF-MS operating parameters were as follows: capillary voltage, $1.0-1.5 \mathrm{kV}$; sample cone voltage, $100-200 \mathrm{~V}$; extraction cone voltage, 50-100 V; source block temperature, $70{ }^{\circ} \mathrm{C}$; source pressure $9.0 \mathrm{mbar}$ (standard $2.0 \mathrm{mbar}$ ), TOF analyzer pressure $1.3 \times 10^{-6} \mathrm{mbar}$ (standard $6.2 \times 10^{-7} \mathrm{mbar}$ ). Spectra were recorded in the positive ion mode and the standard $\mathrm{m} / \mathrm{z}$ range of $200-10,000$ was monitored. The mass spectrometer was calibrated on the singly charged $\mathrm{Cs}_{\mathrm{n}+1} \mathrm{I}_{\mathrm{n}}$ clusters obtained after electrospraying an aqueous cesium iodide solution $(1 \mathrm{mg} / \mathrm{ml})$. Molecular masses of protein and protein-ligand complexes were calculated using a maximum entropy (MaxEnt1) based approach $[19,20]$ incorporated as part of the MassLynx software (MassLynx version 3.5) supplied with the mass spectrometer.

\section{Results and Discussion}

\section{Noncovalent cGMP Binding}

Figure 2 shows the mass spectrum of native PKG sprayed from an aqueous $200 \mathrm{mM}$ ammonium acetate solution at an estimated PKG dimer concentration of $\sim 1-2 \mu \mathrm{M}$. The mass spectrum displays abundant ion signals around $\mathrm{m} / \mathrm{z} 5500-7000$ originating from dimeric PKG I $\alpha$. The molecular weight derived from this charge state envelope is $152,883 \mathrm{Da}$. The theoretical average 
mass of the dimeric protein, calculated from the sequence with acetylated $\mathrm{N}$-termini and without any additional cyclic nucleotides or phosphorylated residues is $152,659 \mathrm{Da}$. A common feature among protein kinase involves activation by phosphorylation of a threonine in the activation loop, which when unphosphorylated may inhibit primarily by blocking the access to the ATP binding site, or by preventing catalytically competent structuring of the active site [1]. Proteolytic digests of PKG analyzed by nanoflow-ESI-TOF-MS/MS revealed that Thr516 within the activation loop is indeed phosphorylated (data not shown). In fact, pure preparations of PKG are known to contain 1,0-1,5 mol phosphate/mol subunit [21]. By taking into account the phosphorylation of this residue, the theoretical mass of dimeric PKG increases to $152,819 \mathrm{Da}$. For now it is assumed that the discrepancy of $\sim 60 \mathrm{Da}$ between the measured and theoretical mass is most likely due to presence of water, buffer components or salts. Each subunit of PKG contains two cyclic nucleotide binding sites with slow and fast dissociation behavior. The amino-terminal binding pocket binds cGMP with an apparent $K_{d}$ of $10 \mathrm{nM}$ while the C-terminal binding pocket binds cGMP with an apparent $K_{d}$ of $100-150 \mathrm{nM}$ $[22,23]$. Addition of free cGMP to the electrospray solution at an estimated cGMP concentration of $3 \mu \mathrm{M}$ results in a direct mass shift of the ion signals of PKG as illustrated for $[\mathrm{M}+25 \mathrm{H}]^{25+}$ ion signal shown in Figure 2a. Deconvolution $[19,20]$ (MaxEnt1) of the charge state envelope indicates that the mass of PKG has increased with $\sim 700 \mathrm{Da}$ (Figure 2b), which would correspond to the binding of two cGMP molecules $\left(\mathrm{M}_{\mathrm{r}} \mathrm{cGMP}=345.2\right)$. Increasing the amount of cGMP added to $10 \mu \mathrm{M}$ results in a further shift of the ion-signals to higher $\mathrm{m} / \mathrm{z}$ ratios. Deconvolution indicates that under these conditions up to four cGMP molecules bind to the dimeric protein, which is in agreement with previous performed biochemical determinations [15].

\section{Ternary PKG-cGMP-AMPPNP/Mn ${ }^{2+}$ Complex}

In order to investigate the binding order and stoichiometry of ATP-binding to PKG and at the same time prevent possible autophosphorylation during the measurement, the non-hydrolyzable ATP analog $\beta, \gamma$-imidoadenosine triphosphate (AMPPNP) was used. Figure 3a shows the mass spectrum of PKG in the presence of $25 \mu \mathrm{M}$ AMPPNP. Deconvolution of the mass spectrum displays binding of one AMPPNP molecule, however the most abundant species is still dimeric PKG without any additional ligands. Addition of $50 \mu \mathrm{M} \mathrm{MnCl}_{2}$ to the spray solution results in a shift of the ion signals to higher $\mathrm{m} / \mathrm{z}$. After deconvolution it appears that the mass of PKG has increased by $1225 \mathrm{Da}$, which corresponds with binding of two times AMPPNP $/ 2 \mathrm{Mn}^{2+}$ (Figure $3 b)$. The mass spectrum of PKG in presence of cGMP $(10 \mu \mathrm{M})$ and AMPPNP $(50 \mu \mathrm{M})$ (Figure 3c) shows solely binding of 4 cGMP molecules. When PKG was electrosprayed with cGMP $(10 \mu \mathrm{M})$, AMPPNP $(50 \mu \mathrm{M})$ and

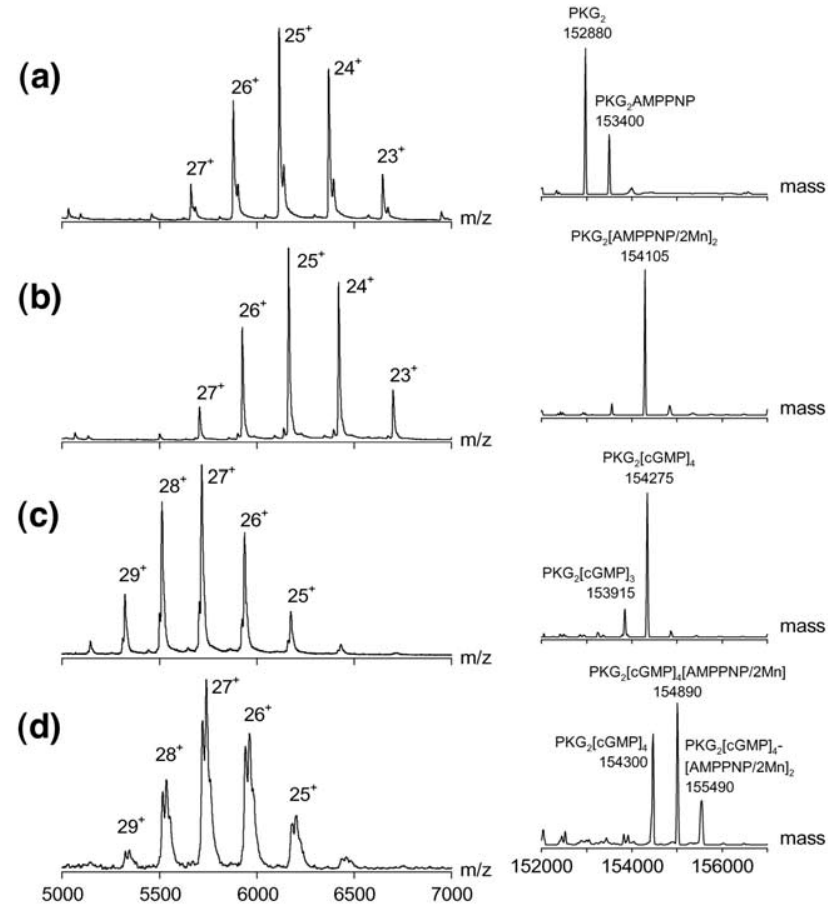

Figure 3. Electrospray ionization mass spectra of PKG at an estimated dimer concentration of $\sim 1-2 \mu \mathrm{M}$ (a) in the presence of $25 \mu \mathrm{M}$ AMPPNP, (b) PKG in the presence of $25 \mu \mathrm{M}$ AMPPNP and $50 \mu \mathrm{M} \mathrm{MnCl}_{2}$, (c) PKG in the presence of $10 \mu \mathrm{M} \mathrm{cGMP}$ and $50 \mu \mathrm{M}$ AMPPNP, and (d) PKG in the presence of $10 \mu \mathrm{M}$ cGMP, $50 \mu \mathrm{M}$ AMPPNP and $100 \mu \mathrm{M} \mathrm{MnCl}{ }_{2}$.

$\mathrm{MnCl}_{2}(100 \mu \mathrm{M})$, binding of two AMPPNP $/ 2 \mathrm{Mn}^{2+}$ is visible, however binding of one AMPPNP $/ 2 \mathrm{Mn}^{2+}$ is the most abundant species and PKG with only four cGMP molecules is also visible (Figure $3 \mathrm{~d}$ ). In another set of experiments, magnesium instead of manganese was added to the spray solution. Although $\mathrm{Mg}^{2+}$ is the physiological cation activator of PKG, complete saturation of PKG with AMPPNP by magnesium could not be observed in the mass spectrum. Figure 4 shows electrospray ionization mass spectra of PKG in the presence of AMPPNP, AMPPNP- $\mathrm{Mn}^{2+}$, and AMPPNP- $\mathrm{Mg}^{2+}$. In the low $\mathrm{m} / \mathrm{z}$ region coordination of $\mathrm{Mg}^{2+}$ and $\mathrm{Mn}^{2+}$ to AMPPNP is clearly visible, however with an increased AMPPNP $(125 \mu \mathrm{M})$ concentration and relatively high magnesium concentration used (1.2 mM) AMPPNP/ $\mathrm{Mg}^{2+}$ complexation to PKG is observed (Figure $4 \mathrm{~b}$ and c), but it is still much less pronounced than with $\mathrm{Mn}^{2+}$.

\section{Limited Proteolysis}

Type I PKGs homo-dimerize through interactions at the N-terminus of each monomer [24]. This section has been designated the dimerization domain on the basis of a leucine/isoleucine zipper motif and for type I $\alpha$ also on the presence of a cysteine residue within. Removal of the N-terminal inhibition and dimerization domain by limited proteolysis completely monomerizes the protein, which will still bind $2 \mathrm{~mol}$ of cGMP/subunit with 
(a)

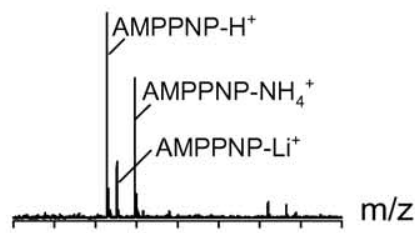

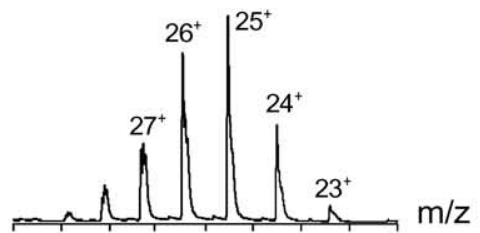

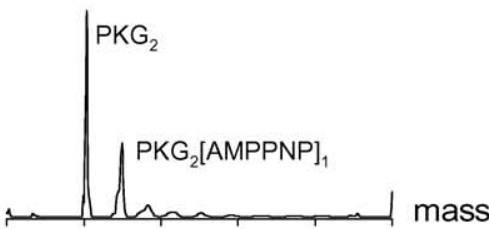

(b)

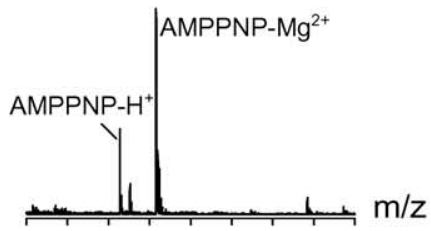

(c)

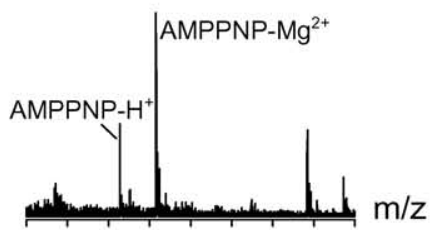

(d)

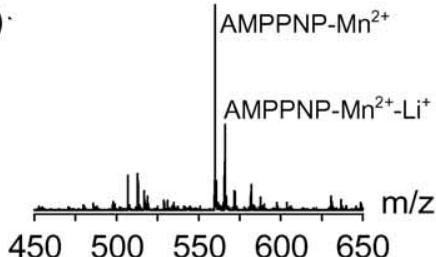

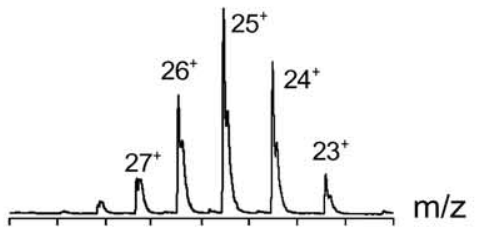

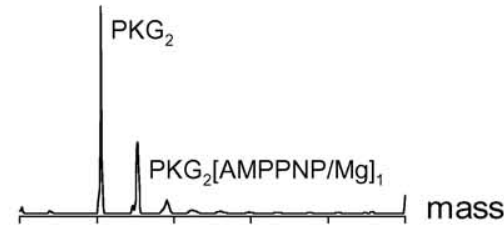

Figure 4. Electrospray ionization mass spectra of PKG at an estimated dimer concentration of $\sim 1-2$ $\mu \mathrm{M}(\mathbf{a})$ in the presence of $25 \mu \mathrm{M}$ AMPPNP, (b) in the presence of $25 \mu \mathrm{M}$ AMPPNP and $1.2 \mathrm{mM}$ $\mathrm{Mg}^{2+}$-acetate, (c) in the presence of $125 \mu \mathrm{M}$ AMPPNP and $1.2 \mathrm{mM} \mathrm{Mg}^{2+}$-acetate, and (d) in the presence of $25 \mu \mathrm{M}$ AMPPNP and $100 \mu \mathrm{M} \mathrm{Mn}^{2+}$-chloride. Mass spectra on the left display the low $\mathrm{m} / \mathrm{z}$ region from 450-650, displaying the unbound or free AMPPNP molecular ion. Mass spectra in the middle display the charge state envelope of dimeric PKG and mass spectra on the right display the corresponding deconvoluted mass spectra.

an unchanged enzymatic activity [16, 25]. Figure 5 shows the mass spectrum of PKG I $\alpha$ before (Figure 5a) and after (Figure 5b) limited proteolysis with chymotrypsin. As already mentioned the mass spectrum of non-proteolyzed protein shows abundant ion peaks around $\mathrm{m} / \mathrm{z} 5500-7000$. Figure $5 \mathrm{~b}$ shows the electrospray ionization mass spectrum of PKG after limited chymotryptic hydrolysis. Two charge state envelopes are clearly visible around $\mathrm{m} / \mathrm{z} 2000$ and 4500 . These two envelopes correspond to the dimeric species of the first $70 \mathrm{~N}$-terminal residues of PKG (measured mass 16430.6 $\mathrm{Da}$, theoretical mass $16430.9 \mathrm{Da}$ considering a disulfide bridge between Cys-42 of each polypeptide chain), and to the large monomer comprising of residues $81-670$ carrying one cAMP molecule (measured mass 67,305 $\mathrm{Da}$, theoretical mass $67,281 \mathrm{Da}$ ). Occasionally, the doubly charged peptide of residues $71-80$ at $\mathrm{m} / \mathrm{z} 638.88$ (theoretical $\mathrm{m} / \mathrm{z} 638.83$ ) could be observed in the low $\mathrm{m} / \mathrm{z}$ region. Limited proteolysis of differentially autophosphorylated protein was performed to gain further in- sight into the overall amount of phosphate incorporation during the autophosphorylation reaction. Figure 6 shows enlarged parts of the electrospray ionization mass spectrum of partially proteolyzed PKG, which was non-, partially-, and highly-autophosphorylated. In the low mass range $(\mathrm{m} / \mathrm{z} 620-700)$ the doubly charged peptide of residues 71-80 displayed a single mass increase of $80 \mathrm{Da}$ in the partially phosphorylated state. Unfortunately, this peptide could not be observed in the highly autophosphorylated sample. The charge state envelope around $\mathrm{m} / \mathrm{z} 2000$, corresponding to the dimeric species of the first 70 residues of PKG, shows multiple increases in mass of $80 \mathrm{Da}$. The $[\mathrm{M}+8 \mathrm{H}]^{8+}$ ion signal of the dimer of residues 1-70 shows no detectable amount of phosphorylation in the non-phosphorylated protein sample. Partially phosphorylated protein contains on average two phosphate moieties within the dimer of the first 70 residues, while highly autophosphorylated protein contains on average four phosphate moieties within the dimer of the first 70 residues and 


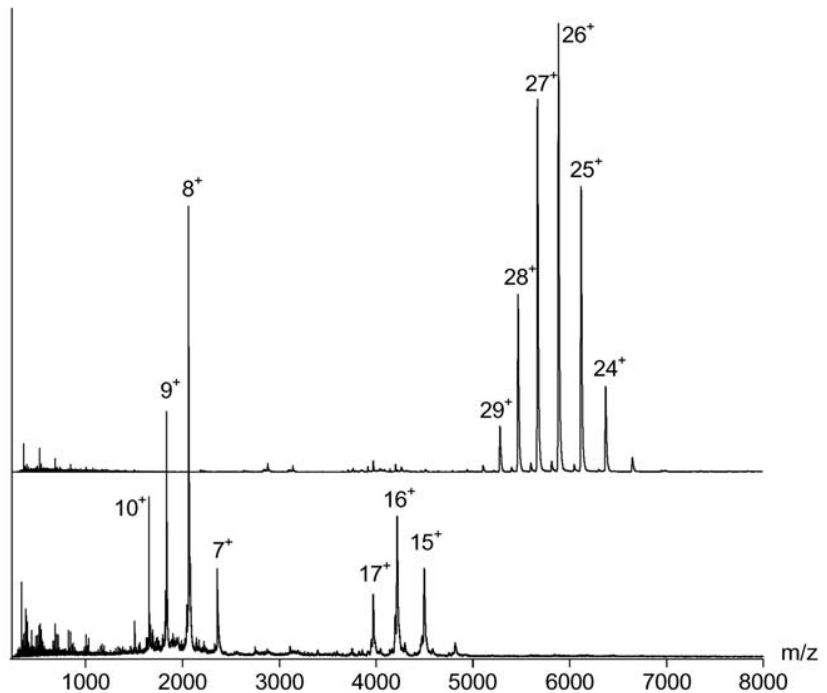

Figure 5. Electrospray ionization mass spectra of native PKG at an estimated dimer concentration of $\sim 1-2 \mu \mathrm{M}$ before (top) and after (bottom) limited proteolysis with chymotrypsin. Around $\mathrm{m} / \mathrm{z}$ $=2000$ ion signals corresponding to the dimer of residues $1-70$ are visible, around $m / z=4000$ ion signals for residues $81-670$ are visible. Occasionally the double charged peptide of residues 71-80 was detectable in the low $\mathrm{m} / \mathrm{z}$ range.

shows a maximum of 6 to 7 phosphorylation sites within this part of PKG. As previously mentioned PKG is already phosphorylated on threonine 516 in nonautophosphorylated protein samples. Additionally, the large monomeric subunit (residues 81-670) shows in the highly autophosphorylated protein sample an additional single mass increase of $80 \mathrm{Da}$, indication that one autophosphorylation site lies within the residues 81-670.

\section{Conclusions}

Activity modulation via phosphorylation is the predominant mechanism of signal transduction in cellular pathways. Phosphorylation and dephosphorylation events are catalyzed by protein kinases and protein phosphatases, respectively. In particular, protein kinases are themselves tightly regulated by phosphorylation or allosteric activation. In this study we have examined the activation properties of PKG using a mass spectrometry based approach. Our nanoflow ESITOF-MS analysis under non-denaturing conditions confirms that PKG mainly occurs as a $153 \mathrm{kDa}$ homodimer and is able to bind four cGMP molecules, which is in agreement with the known stoichiometry. Additionally, nanoflow ESI-TOF-MS data of PKG in complex with AMPPNP $/ \mathrm{Mn}^{2+}$ clearly demonstrates that PKG is able to bind two AMPPNP molecules in an inactive conformation of the protein (i.e., in the absence of cGMP). Moreover, this interaction was only observed in the presence of the divalent metal $\mathrm{Mn}^{2+}$. This observation is in good agreement with the requirement of a divalent metal for binding of ATP in the ATP binding pocket as seen in many crystal structures of protein kinases. In the inactive conformation the autoinhibitory domain within the $\mathrm{N}$-terminal regulatory domain interacts with the substrate-docking domain on the larger lobe of the catalytic core. Our finding could imply that within this inactive conformation the ATP binding pocket is not blocked and AMPPNP or ATP are still able to enter this part of the catalytic domain. In the active conformation (i.e., when cGMP is bound) AMPPNP binding is still observed in the mass spectrum, however, at a higher concentration of AMPPNP and less of the fully saturated product is observed. In the crystal structure of PKA, ATP is bound inside the cleft between the smaller

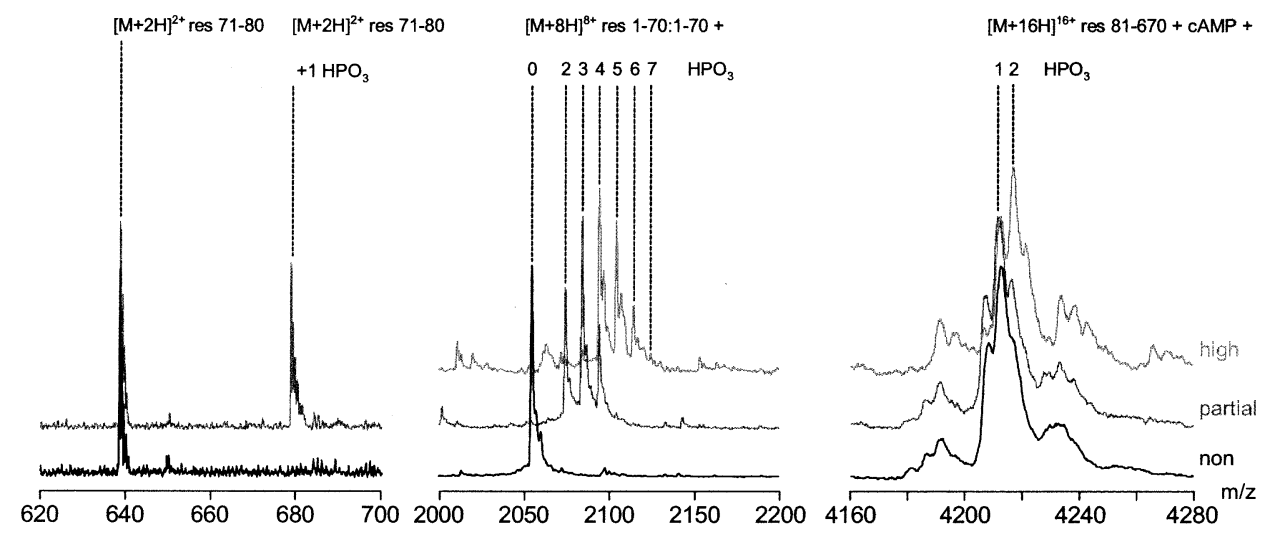

Figure 6. Enlarged parts of the nanoflow ESI mass spectrum of partially proteolyzed PKG I $\alpha$, which was autophosphorylated to different levels. Enlarged parts of the mass spectra are displayed for partially proteolyzed PKG, which was (from top to bottom) non-, partially-, and highly-autophosphorylated. Mass spectra on the left display the low $\mathrm{m} / \mathrm{z}$ region from $\mathrm{m} / \mathrm{z} 620-700$, displaying the double charge molecular ion of residues 71-80. Mass spectra in the middle display the $[\mathrm{M}+8 \mathrm{H}]^{8+}$ of the dimeric N-terminus (residues 1-70). Mass spectra on the right display the $[\mathrm{M}+16 \mathrm{H}]^{16+}$ of the C-terminal residues 81@0017670. The number of phosphate groups present on each of the three proteolytic fragments are listed above each ion signal. 
and larger lobe of the catalytic domain. Opening and closing of this active site cleft serves to position the ATP for catalysis. When PKG is inactive, the interaction between the N-terminal domain and the substratedocking domain might have a stabilizing effect on the flexibility in opening and closing of the active cleft. Hence the obtained complex between AMPPNP $/ \mathrm{Mn}^{2+}$ and PKG might be more stable. In the presence of cGMP, the interaction between the $\mathrm{N}$-terminal domain and the substrate binding site is released. Hence it might well be possible that the two lobes of the catalytic domain receive more degrees of freedom, as a result ATP-binding is less strong and this is somehow reflected in the mass spectrum of PKG in presence of cGMP and AMPPNP $/ \mathrm{Mn}^{2+}$. For PKA, nucleotide binding and the influence of divalent metals have been studied extensively [26-28]. Magnetic resonance studies of the interaction between the catalytic subunit of PKA, nucleotides, and $\mathrm{Mn}^{2+}$ revealed the binding of two $\mathrm{Mn}^{2+}$ ions per molecule PKA. Affinities of the $\mathrm{Mn}^{2+}$ for the two binding sites in a binary PKA-AMPPCP complex were $6-10 \mu \mathrm{M}$ and $50-60 \mu \mathrm{M}$ respectively. Kinetic analysis revealed that the high affinity $\mathrm{Mn}^{2+}$ binding has activation properties, while the lower affinity has an inhibitory effect [26]. In the same study, two binding sites of $\mathrm{Mg}^{2+}$ were detected with apparent much lower binding affinity of $1.6 \mathrm{mM}$ for both sites. The effect of the physiological activator $\mathrm{Mg}^{2+}$ was analogous to those found with $\mathrm{Mn}^{2+}$, however the inhibition by $\mathrm{Mg}^{2+}$ was less pronounced than that observed with $\mathrm{Mn}^{2+}$. Additionally, thermal stability measurements on PKA have shown that MnATP enhances stability stronger than MgATP [29]. These observations made with PKA might explain why in our study no homogenous PKG-[AMPPNP $\left./ \mathrm{Mg}^{2+}\right]_{2}$ complex was observed. Although magnesium seems to bind to AMPPNP as illustrated in Figure $4 \mathrm{~b}$ and c, complete saturation of PKG with AMPPNP/ $/ \mathrm{Mg}^{2+}$ was not achieved. Due to the high homology between the catalytic core between PKA and PKG, it is not unlikely that the affinities for ATP and the divalent metal ions are similar for both protein kinases. This would explain why the complex between PKG and AMPPNP in the presence of magnesium as divalent metal ion is more difficult to saturate than that in the presence of manganese.

Upon cGMP-activation PKG phosphorylates in an autocatalytic manner. In this study limited proteolysis in combination with nanoflow ESI-TOF-MS allowed accurate mass measurement of the $\mathrm{N}$-terminal domain. This introduces a higher level of resolution in the part in which almost all known autophosphorylation events occur [30]. This resolution might not have been present for the intact protein. Partially phosphorylated protein showed a single phosphorylation within residues 7180 , and two phosphorylations within the dimer of residues 1-70. Highly autophosphorylated protein showed one phosphorylation within residues 81-670, and further a higher phosphorylation degree in the
N-terminal 1-70 residues. In conclusions, limited chymotryptic proteolysis of PKG in combination with ESITOF-MS allows a fast and accurate determination of the overall phosphorylation status.

\section{Acknowledgments}

The authors thank Jennifer L. Busch, Jackie D. Corbin, and Sharron $\mathrm{H}$. Francis of the Vanderbilt University, School of Medicine for the preparation of autophosphorylated PKG. They also thank Arjen Scholten and Wolfgang R. G. Dostmann of the University of Vermont for the recombinant PKG samples.

\section{References}

1. Adams, J. A. Kinetic and Catalytic Mechanisms of Protein Kinases. Chem. Rev. 2001, 101(8), 2271-2290.

2. Taylor, S. S.; Buechler, J. A.; Yonemoto, W. cAMP-Dependent Protein Kinase: Framework for a Diverse Family of Regulatory Enzymes. Annu. Rev. Biochem. 1990, 59, 971-1005.

3. Johnson, D. A.; Akamine, P.; Radzio-Andzelm, E.; Madhusudan, M.; Taylor, S. S. Dynamics of cAMP-Dependent Protein Kinase. Chem. Rev. 2001, 101(8), 2243-2270.

4. Francis, S. H.; Corbin, J. D. Cyclic Nucleotide-Dependent Protein Kinases: Intracellular Receptors for cAMP and cGMP Action. Crit. Rev. Clin. Lab. Sci. 1999, 36(4), 275-328.

5. Carvajal, J. A.; Germain, A. M.; Huidobro-Toro, J. P.; Weiner, C. P. Molecular Mechanism of cGMP-Mediated Smooth Muscle Relaxation. J. Cell Physiol. 2000, 184(3), 409-420.

6. Hofmann, F.; Ammendola, A.; Schlossmann, J. Rising Behind NO: cGMP-Dependent Protein Kinases. J. Cell Sci. 2000, 113(Pt 10), 1671-1676

7. Takio, K.; Wade, R. D.; Smith, S. B.; Krebs, E. G.; Walsh, K. A.; Titani, K. Guanosine Cyclic 3',5'-Phosphate Dependent Protein Kinase, a Chimeric Protein Homologous with Two Separate Protein Families. Biochemistry 1984, 23(18), 4207-4218.

8. Francis, S. H.; Poteet-Smith, S. B.; Busch, J. L.; Richie-Jannetta, R.; Corbin, J. D. Mechanisms of Autoinhibition in Cyclic Nucleotide-Dependent Protein Kinases. Front. Biosci. 2002, 7, d580-d592.

9. Pfeifer, A.; Ruth, P.; Dostmann, W.; Sausbier, M.; Klatt, P.; Hofmann, F. Structure and Function of cGMP-Dependent Protein Kinases. Rev. Physiol. Biochem. Pharmacol. 1999, 135, 105-149.

10. Chu, D. M.; Francis, S. H.; Thomas, J. W.; Maksymovitch, E. A.; Fosler, M.; Corbin, J. D. Activation by Autophosphorylation or cGMP Binding Produces a Similar Apparent Conformational Change in cGMP-Dependent Protein Kinase. J. Biol. Chem. 1998, 273(23), 14649-14656.

11. Robinson, C. V.; Chung, E. W.; Kragelund, B. B.; Knudsen, J.; Aplin, R. T.; Poulsen, F. M.; Dobson, C. M. Probing the Nature of Noncovalent Interactions by Mass Spectrometry. A Study of Protein-CoA Ligand Binding and Assembly. J. Am. Chem. Soc. 1996, 118(36), 8646-8653.

12. McCammon, M. G.; Scott, D. J.; Keetch, C. A.; Greene, L. H.; Purkey, H. E.; Petrassi, H. M.; Kelly, J. W.; Robinson, C. V. Screening Transthyretin Amyloid Fibril Inhibitors: Characterization of Novel Multiprotein, Multiligand Complexes by Mass Spectrometry. Structure (Camb), 2002, 10(6), 851-863.

13. Loo, J. A. Studying Noncovalent Protein Complexes by Electrospray Ionization Mass Spectrometry. Mass Spectrom. Rev. 1997, 16(1), 1-23.

14. Loo, J. A. Electrospray Ionization Mass Spectrometry: A Technology for Studying Noncovalent Macromolecular Complexes. Int. J. Mass Spectrom. 2000, 200(1/3), 175-186. 
15. Corbin, J. D.; Doskeland, S. O. Studies of Two Different Intrachain cGMP-Binding Sites of cGMP-Dependent Protein Kinase. J. Biol. Chem. 1983, 258(18), 11391-11397.

16. Monken, C. E.; Gill, G. N. Structural Analysis of cGMPDependent Protein Kinase Using Limited Proteolysis. J. Biol. Chem. 1980, 255(15), 7067-7070.

17. Francis, S. H.; Wolfe, L.; Corbin, J. D. Purification of Type I $\alpha$ and Type I $\beta$ Isozymes and Proteolyzed Type I $\beta$ Monomeric Enzyme of cGMP-Dependent Protein Kinase from Bovine Aorta. Methods Enzymol. 1991, 200, 332-341.

18. Dostmann, W. R.; Taylor, M. S.; Nickl, C. K.; Brayden, J. E.; Frank, R.; Tegge, W. J. Highly Specific, Membrane-Permeant Peptide Blockers of cGMP-Dependent Protein Kinase I $\alpha$ Inhibit NO-Induced Cerebral Dilation. Proc. Natl. Acad. Sci. U.S.A. 2000, 97(26), 14772-14777.

19. Ferrige, A. G.; Seddon, M. J.; Green, B. N.; Jarvis, S. A.; Skilling, J. Disentangling Electrospray Spectra with Maximum-Entropy. Rapid Commun. Mass Spectrom. 1992, 6(11), 707-711.

20. Ferrige, A. G.; Seddon, M. J.; Skilling, J.; Ordsmith, N. The Application of Maxent to High-Resolution Mass Spectrometry. Rapid Commun. Mass Spectrom. 1992, 6(12), 765-770.

21. Hofmann, F.; Flockerzi, V. Characterization of Phosphorylated and Native cGMP-Dependent Protein Kinase. Eur. J. Biochem., 1983, 130(3), 599-603.

22. Hofmann, F.; Gensheimer, H. P.; Gobel, C. cGMP-Dependent Protein Kinase. Autophosphorylation Changes the Characteristics of Binding Site 1. Eur. J. Biochem. 1985, 147(2), 361-365.

23. Reed, R. B.; Sandberg, M.; Jahnsen, T.; Lohmann, S. M.; Francis, S. H.; Corbin, J. D. Fast and Slow Cyclic NucleotideDissociation Sites in cAMP-Dependent Protein Kinase are
Transposed in Type I $\beta$ cGMP-Dependent Protein Kinase. J. Biol. Chem. 1996, 271(29), 17570-17575.

24. Richie-Jannetta, R.; Francis, S. H.; Corbin, J. D. Dimerization of cGMP-Dependent Protein Kinase I $\beta$ is Mediated by an Extensive Amino-Terminal Leucine Zipper Motif, and Dimerization Modulates Enzyme Function. J. Biol. Chem. 2003, 278(50), 50070-50079.

25. Wolfe, L.; Francis, S. H.; Corbin, J. D. Properties of a cGMPDependent Monomeric Protein Kinase from Bovine Aorta. J. Biol. Chem. 1989, 264(7), 4157-4162.

26. Armstrong, R. N.; Kondo, H.; Granot, J.; Kaiser, E. T.; Mildvan, A. S. Magnetic Resonance and Kinetic Studies of the Manganese(II) Ion and Substrate Complexes of the Catalytic Subunit of Adenosine 3' $5^{\prime}$-Monophosphate Sependent Protein Kinase from Bovine Heart. Biochemistry 1979, 18(7), 1230-1238.

27. Adams, J. A.; Taylor, S. S. Divalent Metal Ions Influence Catalysis and Active-Site Accessibility in the cAMP-Dependent Protein Kinase. Protein Sci. 1993, 2(12), 2177-2186.

28. Bhatnagar, D.; Roskoski, R., Jr.; Rosendahl, M. S.; Leonard, N. J. Adenosine Cyclic 3',5'-Monophosphate Dependent Protein Kinase: A New Fluorescence Displacement Titration Technique for Characterizing the Nucleotide Binding Site on the Catalytic Subunit. Biochemistry 1983, 22(26), 63106317.

29. Herberg, F. W.; Doyle, M. L.; Cox, S.; Taylor, S. S. Dissection of the Nucleotide and Metal-Phosphate Binding Sites in cAMPDependent Protein Kinase. Biochemistry 1999, 38(19), 63526360.

30. Aitken, A.; Hemmings, B. A.; Hofmann, F. Identification of the Residues on Cyclic GMP-Dependent Protein Kinase that are Autophosphorylated in the Presence of Cyclic AMP and Cyclic GMP. Biochim. Biophys. Acta 1984, 790(3), 219-225. 